\title{
Infant Formula Intolerance
}

National Cancer Institute

\section{Source}

National Cancer Institute. Infant Formula Intolerance. NCI Thesaurus. Code C114928.

Inability to effectively digest or absorb the components of breast milk substitutes.

Symptoms may include emesis, abdominal distension or diarrhea. 